\title{
An Information-Theoretic Approach to Accelerated Simulation of Hybrid-ARQ Systems
}

\author{
Matthew C. Valenti \\ Lane Department of Computer Science and Electrical Engineering \\ West Virginia University, Morgantown, WV, U.S.A. \\ Email: valenti@ieee.org
}

\begin{abstract}
In order to reduce the long runtimes required to simulate hybrid automatic repeat-request (ARQ) systems, such as high-speed downlink packet access (HSDPA), we propose an information-theoretic approach to accelerate the simulation while still maintaining its fidelity. The approach is based on the concept of information-outage probability, and involves the computation of the mutual information between the code bits transmitted over the channel and the bit log-likelihood ratio coming out of the demodulator. Because the mutual information is measured at the demodulator output, the metric naturally accounts for the parameters of the channel and modulation as well as the length of the codeword. An information outage is declared whenever the measured mutual information is below a threshold (the code rate), and the frequency of simulated information outages provides an accurate prediction of the rate that the turbo decoder fails. Because the prediction is sufficiently accurate, it is possible to run the simulation without actually decoding the turbo code. The result is a 10-30 fold speedup in simulation runtime, while the predicted frame error rate and throughput results are still within 1-2 dB of the values obtained with a more accurate (though lengthier) simulation that decodes each turbo codeword.
\end{abstract}

\section{INTRODUCTION}

High-speed downlink packet access (HSDPA) is a part of the UMTS suite of third-generation (3G) cellular standards, and has become a widely adopted technology for achieving highrate downlink data transmissions in a cellular environment [1] [3]. HSDPA is a fairly sophisticated transmission technology, and features the use of turbo coding, multiple orthogonal spreading codes, and hybrid-ARQ along with QPSK or 16QAM modulation. Because of the turbo code, full simulation of the system is computationally intensive. The lengthy simulation runtime is especially a problem when a wide variety of parameters are considered, and hence, many simulations must be executed.

If only AWGN channels are considered, then the only parameters that need to be adjusted are those that relate to the coding and modulation, and thus the number of simulations required for a typical application is manageable. However, if a more sophisticated channel is considered, then a wide variety of parameters related to the channel must also be adjusted. When both the HSDPA parameters and the channel parameters must be adjusted, then the total number of simulations can become extraordinarily large, and a method to accelerate the simulation is desirable.

This work was supported in part by the National Science Foundation under Award No. CNS-0750821.
In this paper, we propose a methodology for performing rapid simulations of HSDPA by building upon the concept of information-outage probability [4]-[6]. This technique bypasses the turbo decoder, and as a consequence can speed up simulation by more than an order of magnitude. However, the results are able to accurately predict throughput and frame error rate to within $1-2 \mathrm{~dB}$ of a simulation that actually performs turbo decoding. In contrast with prior work on information outage, this methodology explicitly accounts for the frame length of the code as well as the parameters of the frequency-selective channel.

Our approach is not meant to be a replacement for a full simulation that includes the turbo decoder. However, it enables a large initial simulation campaign to be run very quickly. On the basis of the initial simulation campaign, poor designs can be quickly identified and discarded, while better designs could be re-simulated more accurately (i.e., by using the turbo decoder in the second set of simulations).

The remainder of this paper is organized as follows. Section II provides an overview of the HSDPA standard. The information-outage probability concept is described in Section III, while Section IV gives a brief example of its application to an AWGN channel. The frequency-selective channel model is given in Section V, while Section VI applies the informationoutage probability concept to an HSDPA system signaling over a frequency-selective channel with receive antenna diversity. Finally, Section VII concludes the paper.

\section{OVERVIEW OF HSDPA}

In HSDPA, time is segmented into $2 \mathrm{msec}$ long frames called transmission time intervals (TTI's). During each TTI, one data message is turbo encoded, modulated, and spread. Prior to turbo encoding, a 24 bit cyclic redundancy check (CRC) is appended to the data. The CRC is used at the receiver to detect uncorrected errors. The turbo encoder has an internal interleaver that may be set to any length between 40 and 5114 message bits, inclusive. If the CRC-encoded message is longer than 5114 bits, then it must be segmented into multiple blocks of equal length.

Each length $K$ block is encoded by the turbo encoder to produce a turbo codeword of length $3 K+12$ (the final twelve bits are due to the tail). The overall base rate is thus $K /(3 K+12)$, though other rates may be desired. The code rate may be adjusted through a process called rate matching, which 
achieves higher rates by puncturing code bits and lower rates by repeating code bits. The rate-matched code blocks are then modulated using either QPSK or 16-QAM modulation and spread using length 16 Walsh codes at a chip rate of $R_{c}=3.84$ Mchips/second. Because Walsh codes are orthogonal, their use effectively splits the channel into 16 subchannels, called physical channels. One of the physical channels is used as a pilot channel to facilitate coherent reception and channel estimation. Of the remaining 15 physical channels, any or all may be used to transmit the turbo encoded blocks. Let $P$ denote the number of physical channels used per TTI, where $1 \leq P \leq 15$.

The symbol rate of each physical subchannel is $R_{s}=$ $R_{c} / 16=240$ kbaud. Thus, the overall symbol rate for the link is $240 P$ kbaud. During a 2 msec TTI, there are 480 symbols per physical channel, which corresponds to 960 code bits when QPSK is used or 1920 code bits when 16-QAM is used. The maximum rate that code bits may be transmitted when 16-QAM is used with all 15 physical channels is $15(1920) /\left(2 \times 10^{-3}\right)=14.4$ Mbps. The actual data transmission rate will be lower, in accordance with the rate of the rate-matched turbo code and the number of physical channels used. The actual maximum data transmission rate can be found by dividing the number of data bits encoded per TTI by 2 msec. The spread physical channels are summed and then passed the RF modulator. Prior to RF modulation, the spread and modulated symbols are passed through a raised-cosine rolloff (RC) chip-shaping filter with rolloff factor $\alpha=0.22$, resulting in a radiated $\mathrm{RF}$ bandwidth of $R_{c}(1+\alpha)=4.7 \mathrm{MHz}$.

HSDPA has the option of supporting hybrid-ARQ. If hybridARQ is enabled and the receiver is unable to correct a received TTI, then it will request a retransmission by sending a negative acknowledgement (NACK) signal back to the transmitter. The receiver will be able to determine that it did not properly correct the received TTI by examining the CRC error detection code that was appended prior to transmission. If the second transmission fails, a third could be requested, and if the third fails, then a fourth could be requested. While it could be possible to attempt more than four transmissions, the reference designs in the standard [2] limit the number of hybrid-ARQ attempts to four.

When a retransmission is requested, the system can respond by either sending the same packet again or sending additional parity bits. When the same packet is retransmitted, the system is in the Chase Combining (CC) mode of operation. If, instead, a set of additional parity bits is sent, the system is in the Incremental Redundancy (IR) mode of operation. IR is implemented by configuring the rate-matching processor to puncture a different set of bits than was punctured during the initial transmission.

The manner that the symbols in the constellation are labeled with code bits is called a symbol-labeling map. While the performance of QPSK is insensitive to the symbol-labeling map, the performance of 16-QAM is very sensitive to the mapping. In a QAM constellation, the interior points have four neighbors and are more vulnerable to noise than the corner

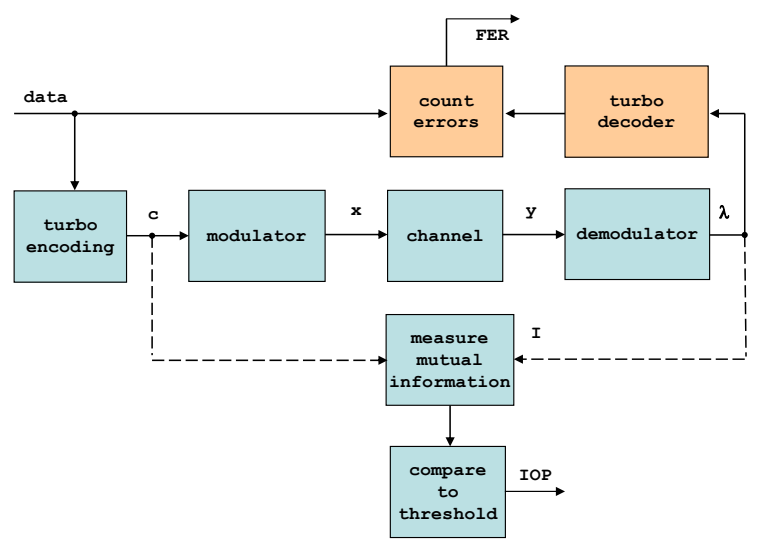

Fig. 1. The information-outage concept. Both conventional and informationoutage simulators follow the path in the second row. The conventional simulator follows the path on the top row of the diagram, while the informationoutage simulator follows the path on the bottom two rows of the diagram.

points which have only two neighbors. Thus, the raw error rate of the different symbols will be unequal. When IR is used with 16-QAM, additional diversity is achieved through constellation rearrangement, which involves the use of a different 16QAM symbol-labeling map each time an IR retransmission is request. The justification for this strategy is that if the symbol-labeling map used for the initial transmission was "bad" for the particular codeword and channel realization, then the rearrangement may produce a symbol-labeling map that is more suitable during the next attempt.

Due to propagation and processing delays, there will be a non-negligible delay from the time that a TTI is transmitted to when the ACK/NACK is received over the return channel. To compensate for this delay, HSDPA allows multiple hybridARQ processes to run concurrently. For instance, in the reference designs in [2], six hybrid-ARQ processes run concurrently. While the transmitter is waiting for the ACK/NACK to be returned for a particular TTI, it can service the other five hybrid-ARQ processes in a round-robin fashion. This allows a delay of $10 \mathrm{msec}$ from the end of the transmission of the TTI until it must be retransmitted. This delay must account for the round-trip propagation delay, processing time at the receiver, and also time for the transmitter to process the ACK/NACK message sent over the return channel.

\section{INFORMATION-OUTAGE ANALYSIS}

While the analysis of a few link designs over an AWGN channel can be achieved by running complete simulations, such simulations become prohibitively complex when one wants to investigate a wide variety of link designs over a wide range of fading conditions. In order to accelerate the simulations, a reduced-complexity simulation methodology based on the concept of information-outage can be used.

Fig. 1 illustrates the information-outage concept. In both conventional and information-outage simulations, the four blocks in the middle row of the diagram are implemented. This requires that the data be encoded by a rate $R$ turbo encoder, modulated, sent over the channel, and demodulated. 
The output of the demodulator is a sequence of log-likelihood ratios (LLRs) $\left\{\lambda_{j}, 1 \leq j \leq n\right\}$ of the code bits. In a conventional, full-complexity simulation, the LLRs are passed through a turbo decoder, the output of the decoder is compared with the data, and an error count made. In an informationoutage simulation, the turbo decoder is bypassed, and instead the mutual information between the code bits and bit LLRs is computed.

The demodulator calculates the LLR of each code bit $c_{j}$ given the corresponding received symbol $y$ using [7]

$$
\lambda_{j}=\log \frac{\operatorname{Pr}\left[c_{j}=1 \mid y\right]}{\operatorname{Pr}\left[c_{j}=0 \mid y\right]}=\log \frac{\sum_{x^{\prime} \in \mathcal{S}_{j}^{(1)}} p\left(y \mid x^{\prime}\right)}{\sum_{x^{\prime} \in \mathcal{S}_{j}^{(0)}} p\left(y \mid x^{\prime}\right)}
$$

where $\mathcal{S}_{j}^{(1)}$ is the set containing the $M / 2$ symbols in the constellation whose $j^{\text {th }}$ bit is labeled with a $1, \mathcal{S}_{j}^{(0)}$ is the set containing the $M / 2$ symbols whose $j^{\text {th }}$ bit is labeled with a 0 , and $p\left(y \mid x^{\prime}\right)$ is the probability that signal $y$ is received when symbol $x^{\prime}$ is sent.

Once the LLR is calculated, the mutual information $I_{j}$ between the $j^{\text {th }}$ code bit and its corresponding LLR $\lambda_{j}$ is found by [7]

$$
\begin{aligned}
I_{j} & =1+\log _{2} p\left(c_{j} \mid \lambda_{j}\right) \\
& =1+\frac{\max *\left(0, \lambda_{j}(-1)^{c_{j}}\right)}{\ln (2)}
\end{aligned}
$$

where the $\max *(\cdot, \cdot)$ operator is defined as [8]

$$
\begin{aligned}
\max *(x, y) & =\log \left(e^{x}+e^{y}\right) \\
& =\max (x, y)+\log \left(1+e^{-|y-x|}\right) .
\end{aligned}
$$

Once the mutual information is found for each of the $n$ code bits, the average mutual information $\bar{I}$ for the codeword can be found by calculating the sample mean

$$
\bar{I}=\frac{1}{n} \sum_{j=1}^{n} I_{j} .
$$

An information-outage occurs when the average mutual information of a codeword is below the code rate $R$. Thus the information-outage probability $(I O P)$ is

$$
P_{0}=\operatorname{Pr}[\bar{I} \leq R] .
$$

The main benefit of the information-outage methodology is speed of execution. Because the decoder is the dominant part of the simulation, information-outage simulations can run between 10 and 30 times faster than simulations that require decoding.

The information-outage methodology is motivated by the fact that turbo codes are capacity approaching. The mutual information can be thought of as the "instantaneous" capacity for the particular codeword and channel realization. If the mutual information is lower than the code rate, the decoder will almost always fail since it is not possible for any code to perform better than capacity. Note that because turbo codes do not fully achieve capacity, it is possible that the decoder could still fail even if the channel is not in an outage. Thus, the IOP

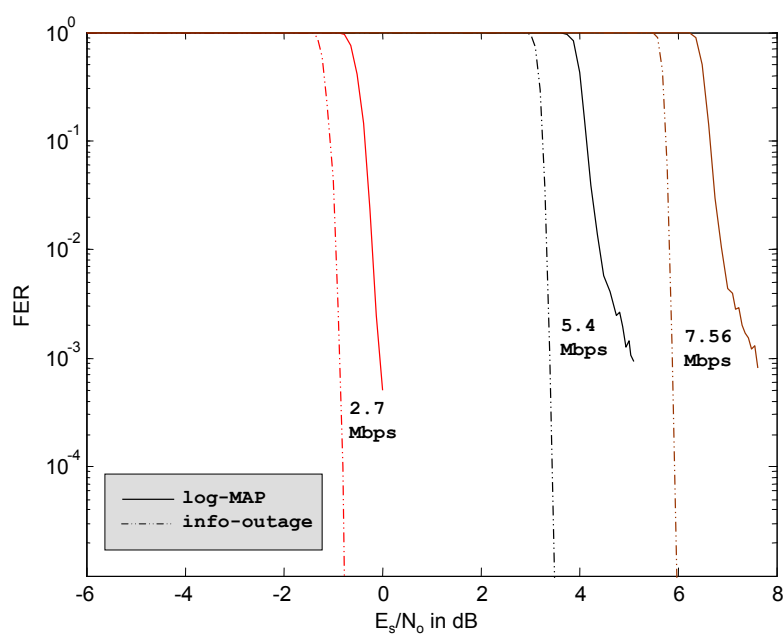

Fig. 2. FER with log-MAP turbo decoding and information-outage probability of three link configurations considered for the AWGN channel.

can be regarded as a practical lower limit on the frame error rate (FER) of the actual turbo coded system. However, the difference between FER and IOP is often negligible, making the IOP an effective estimate of the performance of a particular link configuration or channel condition.

\section{Performance in AWGN}

As an illustration of the information-outage concept, we simulated HSDPA over a simple AWGN channel. We considered three link configurations, capable of achieving rates of 2.7 Mbps, 5.4 Mbps, and 7.56 Mbps. The 2.7 Mbps link uses QPSK modulation and $P=14$ physical channels, while the 5.4 and $7.56 \mathrm{Mbps}$ links uses 16-QAM modulation and $P=15$ physical channels. The code rates after rate matching are $0.4018,0.375$, and 0.525 for the $2.7,5.4$, and $7.56 \mathrm{Mbps}$ links, respectively. None of the links use hybrid-ARQ. Fig. 2 shows two curves for each link configuration: (1) The FER with 14 iterations of log-MAP decoding [8], and (2) The IOP obtained using the methodology described in Section III. Both curves are shown as a function of $\mathcal{E}_{s} / N_{0}$, which is in the ratio of modulated symbol energy to one-sided noise-spectral density. As can be seen from the figure, the IOP curve is consistently about $1 \mathrm{~dB}$ better than the FER, and thus we may conclude that the IOP is an optimistic, but accurate approximation for the FER

\section{Frequency-Selective Channel Model}

A frequency-selective channel is characterized by its powerdelay profile (PDP). While there are many different models available, we use the one described in [9], which has a PDP that takes on the form

$$
S_{\tau}(\tau)=2 \pi f_{0} e^{-2 \pi f_{0} \tau} u(\tau)
$$

where $f_{0}$ is the frequency-selective bandwidth of the channel, $u(\tau)$ is the unit-step function, and $\tau$ is the excess delay. In order to simulate the system, (6) is sampled with a sample 
period $\Delta_{s}$, resulting in

$$
S_{\tau}(\tau)=\sum_{k=0}^{K-1} P_{k} \delta\left(\tau-\tau_{k}\right)
$$

where $K$ is the required number of delay samples, $\tau_{k}=k \Delta_{s}$ is the delay of the $k^{t h}$ sample, and $P_{k}$ is the mean signal power, found by integrating (6) over one sample period,

$$
P_{k}=e^{-2 \pi f_{0} \tau_{k}}-e^{-2 \pi f_{0} \tau_{k+1}} .
$$

The value of $K$ is obtained by finding the minimum number of samples which contain the fraction $P_{\tau}$ of the total received signal power,

$$
K=1-\left\lceil\frac{\ln \left(1-P_{\tau}\right)}{2 \pi f_{0} \Delta_{s}}\right\rceil .
$$

At time $t$, the impulse response of the channel with sampled delay profile (7) is

$$
h(t ; \tau)=\sum_{k=0}^{K-1} \sqrt{P_{k}} c_{k}(t) \delta\left(\tau-\tau_{k}\right)
$$

where $c_{k}(t)$ are unit power complex Gaussian processes. According to the Wide-Sense Stationary Uncorrelated Scattering (WSSUS) model [10], the $K$ multi-path components in (10) are mutually uncorrelated processes with identical power spectrums. While in the general case of Rician fading, the $c_{k}(t)$ could each have a nonzero mean, we assume that the $c_{k}(t)$ are zero-mean, in which case the fading is Rayleigh. Furthermore, the $c_{k}(t)$ are correlated such that they have power spectral density

$$
S(f)=\frac{4 \tau_{R C}}{1+2\left(2 \pi \tau_{R C} f\right)^{2}+\left(2 \pi \tau_{R C} f\right)^{4}}
$$

where $\tau_{R C}=\tau_{0} / 2.1416193$ and $\tau_{0}$ is the signal decorrelation time, defined to be the value of the autocorrelation function $R(\tau)=\mathcal{F}^{-1}\{S(f)\}$ satisfying $R\left(\tau_{0}\right)=e^{-1}$ [11]. A fading process with this power spectral density is easily implemented by passing white Gaussian noise through a two-pole digital filter [9].

In HSDPA, the transmitted signal is passed through a raisedcosine rolloff (RC) chip-shaping filter, which has impulse response [12]

$$
x_{R C}(\tau)=\operatorname{sinc}\left(\frac{\tau}{T_{c}}\right)\left[\frac{\cos \left(\alpha \pi \tau / T_{c}\right)}{\left(1-2 \alpha \tau / T_{c}\right)^{2}}\right]
$$

where $\alpha=0.22$ is specified in [3].

The overall channel is the cascade of the pulse-shaping filter, with impulse response (12), and the wireless channel, with impulse response (10). By convolving these two impulse responses, the impulse response of the equivalent channel $f(t ; \tau)$ is found to be

$$
\begin{aligned}
f(t ; \tau) & =x_{R C}(\tau) * h(t ; \tau) \\
& =x_{R C}(\tau) * \sum_{k=0}^{K-1} \sqrt{P_{k}} c_{k}(t) \delta\left(\tau-\tau_{k}\right) \\
& =\sum_{k=0}^{K-1} \sqrt{P_{k}} c_{k}(t) x_{R C}\left(\tau-\tau_{k}\right)
\end{aligned}
$$

where the last step assumes that $c_{k}(t)$ changes slowly with respect to the chip period $T_{c}$.

The delayed terms in (13) indicate the presence of intersymbol-interference (ISI). If left unmitigated, the ISI will seriously degrade signal quality. However, if processed appropriately, the frequency-selectivity of the channel can be exploited as a form of diversity. The received signal can be processed by either passing it through an equalizer or a rake receiver. We assume that a rake receiver is used and that signal components separated by at least one chip period $T_{c}$ can be resolved. Note that this assumption may be invalidated by the fact that the Walsh codes used by HSDPA do not have good autocorrelation properties and thus there will be significant ISI even after rake reception. If his is the case, then an equalizer should be used in place of a rake receiver.

Let $L$ be the number of fingers in the rake receiver. The channel gain $g_{\ell}(t)$ of the $\ell^{t h}$ finger, $0 \leq \ell \leq L-1$, is found by passing the received signal through a chip-matched filter and sampling the output with a delay of $\ell T_{c}$. The resulting complex channel gain seen by the $\ell^{t h}$ finger is

$$
\begin{aligned}
g_{\ell}(t) & =f\left(t ; \ell T_{c}\right) \\
& =\sum_{k=0}^{K-1} \sqrt{P_{k}} c_{k}(t) x_{R C}\left(\ell T_{c}-\tau_{k}\right) .
\end{aligned}
$$

Even though the $K$ individual processes $c_{k}(t)$ are mutually independent, the $L$ fingers of the rake receiver are not due to the bandlimiting effects of the chip-shaping filter.

The output of the $L$ fingers are maximal-ratio combined (MRC), resulting in a real-valued channel amplitude gain

$$
a_{M R C}(t)=\sqrt{\sum_{\ell=0}^{L-1}\left|g_{\ell}(t)\right|^{2}} .
$$

The despread and MRC-combined signal is sampled at the symbol rate $R_{s}$. After sampling, the channel amplitude gain of the $i^{\text {th }}$ symbol becomes

$$
\begin{aligned}
a_{M R C}[i] & =a_{M R C}\left(i T_{s}\right) \\
& =\sqrt{\sum_{l=0}^{L-1}\left|\sum_{k=0}^{K-1} \sqrt{P_{k}} c_{k}[i] x_{R C}\left(l T_{c}-\tau_{k}\right)\right|^{2}}
\end{aligned}
$$

where $c_{k}[i]$ is the $i^{t h}$ sample of the process $c_{k}(t)$ when sampled at the symbol rate.

To provide additional diversity, multiple antennas may be used at the receiver. Let $Q$ be the number of antennas at the receiver, which are assumed to be spaced sufficiently far apart that the fading seen by each is statistically independent. Because the channels are independent, each antenna is connected to its own rake receiver and demodulates the signal, producing LLR's of the code bits using (1). The LLRs from the $Q$ antennas are then combined, which according to the laws of LLR arithmetic requires that they be simply added, forming the overall LLR

$$
\lambda_{j}=\sum_{q=0}^{Q-1} \lambda_{j}^{(q)}
$$


where $\lambda_{j}^{(q)}$ is the LLR of the $j^{\text {th }}$ code bit at the output of the $q^{t h}$ antenna's demodulator. The combined LLR (17) is then passed through the turbo decoder in the usual way. Alternatively, if an information outage analysis is being performed, then the mutual information is measured between the input codeword and the LLR given by (17).

\section{Prediction of Performance in FAding}

We now consider the performance of HSDPA over the frequency-selective fading channel described in Section V. To help overcome fading and provide additional time diversity, the downlink is operated using hybrid-ARQ with incremental redundancy (IR). Four link designs that use hybrid-ARQ were considered. All four are reference configurations found in [2], where they are referred to as H-Set-3-QPSK, H-Set-3-QAM, $H$-Set-6-QPSK, and H-Set-6-QAM. For the sake of brevity, we refer to these modulation and coding schemes as MCS 1, MCS 2, MCS 3, and MCS 4, respectively. These four schemes can attain maximum data rates of $1.601 \mathrm{Mbps}, 2.332 \mathrm{Mbps}, 3.219$ Mbps, and 4.689 Mbps, respectively.

To allow for a long round-trip propagation time for the feedback signal (positive/negative ACK) to return to the transmitter, several hybrid-ARQ processes may be run in parallel and serviced in a round-robin fashion. The reference HSDPA link configurations in [2] call for 6 parallel hybrid-ARQ processes, which is sufficient for up to $1,500 \mathrm{~km}$ transmission distance (less if processing delays are taken into account). This is more than enough distance for cellular application, but other applications (e.g. communication with aircraft) could require longer distances, which can be accommodated by simply using more parallel hybrid-ARQ processes. The use of multiple hybrid-ARQ processes has the side benefit of making the duration of a single codeword quite long (when retransmitted), which provides a very high level of time diversity. Such time diversity is especially beneficial if the signal decorrelation time is extremely long, as may happen when the relative velocity between transmitter and receiver is low.

The performance of all four schemes was evaluated for a variable number of receive antennas, number of rake receiver fingers, normalized decorrelation times, and normalized frequency-selective bandwidths. The channel simulator was run with parameters $\Delta_{s}=T_{c} / 2$ and $P_{\tau}=0.99$. Because the wide range of parameters required a large number of simulations, the information-outage concept described in Section III was used so that all simulations could be completed in a reasonable amount of time. For select simulations, the FER was also obtained by including the turbo decoder in the simulation. Performance was assessed not only in terms of IOP and FER, but also in terms of throughput, which is the expected data rate when the hybrid-ARQ retransmissions are taken into account [13]. The throughput of the 4 MCS's is shown in Fig. 3 for the case of 3 receive antennas, a 4 finger rake receiver, normalized frequency-selective bandwidth $f_{0} / R_{c}=0.1$, and normalized signal decorrelation time $\tau_{0} / T_{s}=100$. For each MCS, there are two curves. The more optimistic of the two curves (labeled "info-out.") was

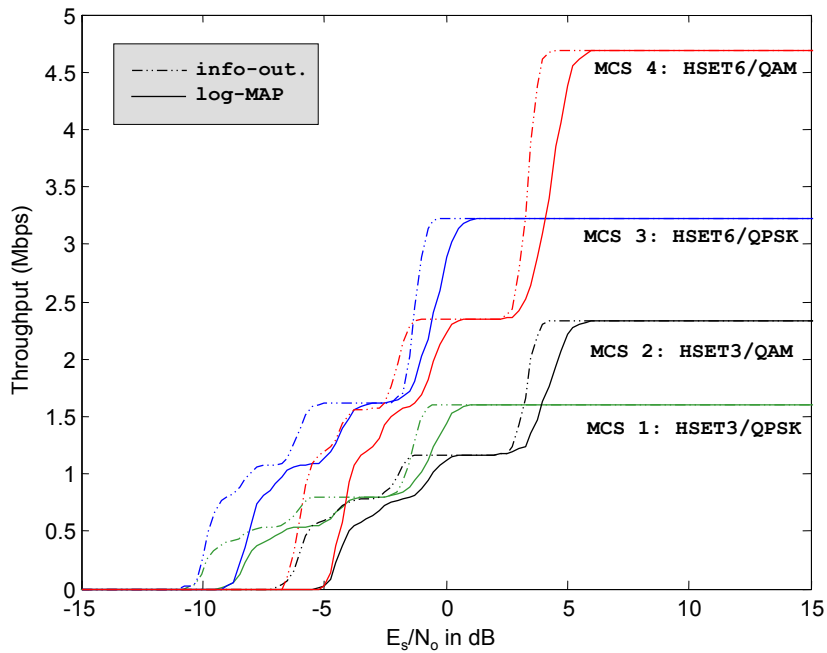

Fig. 3. Throughput of four modulation and coding (MCS) schemes over a frequency-selective fading channel. The throughput was computed using the information-outage probability (dashed lines) and using the FER when performing log-MAP turbo decoding (solid lines).

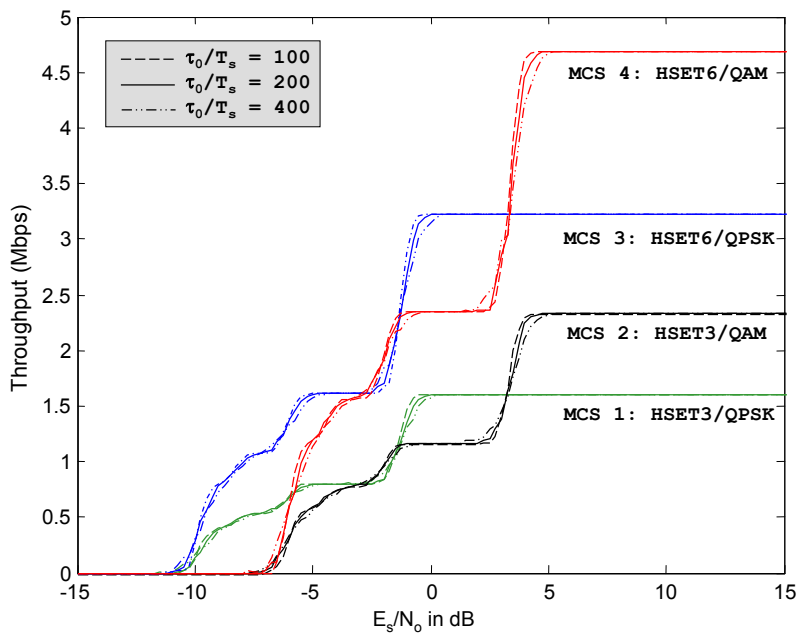

Fig. 4. Influence of the signal decorrelation time on the performance of the four link configurations. The throughput was found using the informationoutage probability.

found by using the information-outage methodology. The less optimistic curve (labeled "log-MAP") was found by simulating the FER of the actual turbo code with log-MAP decoding. As can be seen, the IOP-based method is indeed a good predictor of performance, though because they are not exact it is recommended that a true FER simulation be performed with the turbo code prior to drawing final conclusions.

Having established the usefulness of information-outage probability, we may now use it to quickly generate results that can give some insight into general system behavior. For instance, Fig. 4 shows the effect of varying the signal decorrelation time. After fixing the frequency-selective bandwidth to $f_{0} / R_{c}=0.1$, the number of fingers to $L=4$, and the number of receive antennas to $Q=3$, the normalized signal decorrelation time $\tau_{0} / T_{s}$ was varied. The figure shows results for all four MCS schemes and for three normalized decorrelation times, $\tau_{0} / T_{s}=100,200,400$. The longest absolute decorrelation time was $1.67 \mathrm{msec}$, which is almost an entire 


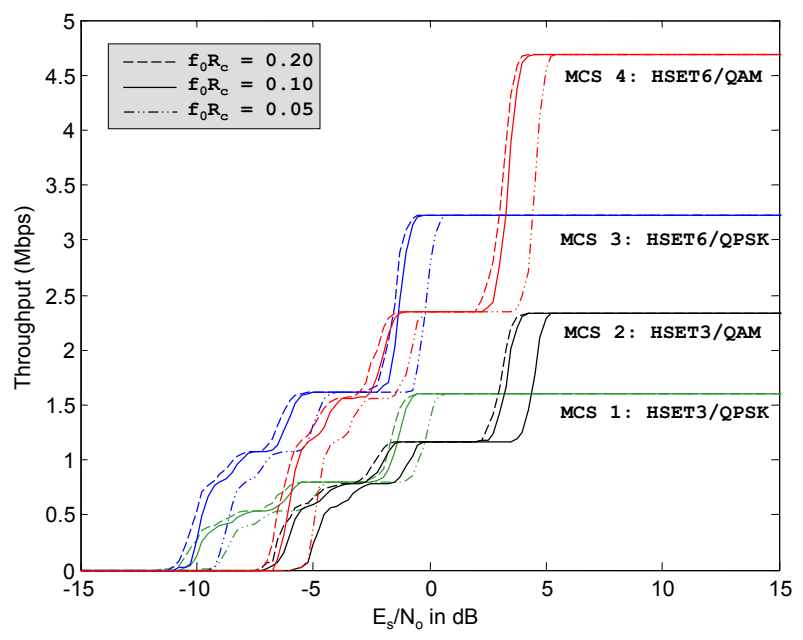

Fig. 5. Influence of the frequency selective bandwidth on the performance of the four link configurations. The throughput was found using the informationoutage probability.

TTI. While it is possible for the channel to produce longer decorrelation times, anything longer than one TTI will be mitigated through the use of multiple hybrid-ARQ processes. For instance, if the absolute decorrelation time is on the order of a TTI $(2 \mathrm{msec})$, then it is likely that if any part of the TTI is in a fade, then the entire TTI will be in a fade. This will trigger a retransmission. However, due to the use of multiple hybridARQ processes that are serviced in a round-robin fashion, the retransmission will not occur until some time later. For instance, with 6 hybrid-ARQ processes, there will be a 5 TTI gap between the end of the first transmission and the start of the next transmission for the same hybrid-ARQ process. This long gap allows the system to handle signal decorrelation times on the order of $10 \mathrm{msec}$. The system can be made more robust against even longer decorrelation times by increasing the number of parallel hybrid-ARQ processes, though this will increase the latency and required buffer sizes.

Fig. 5 shows the effect of varying the frequency-selective bandwidth. After fixing the signal decorrelation time to $\tau_{0} / T_{s}=100$, the number of fingers to $L=4$, and the number of receive antennas to $Q=3$, the normalized frequency-selective bandwidth was varied. The figure shows results for all four MCS schemes and for three normalized frequency-selective bandwidth, $f_{0} / R_{c}=0.05,0.1,0.2$. It can be seen that performance improves with increasing $f_{0} / R_{c}$. For instance, when $f_{0} / R_{c}$ is increased from 0.1 to 0.2 , the system shows an improvement of about $0.5 \mathrm{~dB}$. On the other hand when $f_{0} / R_{c}$ is decreased from 0.1 to 0.05 , there is a loss of about $2 \mathrm{~dB}$. This loss can be attributed to the channel becoming more dispersive as the frequency-selective bandwidth becomes narrower. For instance, from equation (9), there will be $K=16$ taps in the half-chip spaced delay-line model when $f_{0} / R c=0.1$, but when $f_{0} / R_{c}$ is decreased to 0.05 , the number of taps increases to $K=31$. While the 4finger rake receiver is able to capture most of the received signal energy in the $f_{0} / R_{c}=0.1$ case, much of the energy in the $f_{0} / R_{c}=0.05$ case is received after delay $\tau=3 T_{c}$ and therefore is not captured by the rake receiver. Apparently, the solution to handling channels with narrower frequencyselective bandwidths is to use more fingers in the rake receiver.

\section{CONCLUSIONS}

By using the concept of information-outage probability, it is possible to rapidly speed up the simulation of complex transmission technologies, such as HSDPA. The informationoutage probability requires a measurement of the mutual information between the transmitted code bits and log-likelihood ratios available at the output of the demodulator. These values are already available in most simulators, so the amount of modification required to adopt this methodology is quite minimal. Because the mutual information is not measured until the output of the demodulator, the parameters of the channel and modulation are taken into account by the simulation. While the turbo decoder is not used, bits are still turbo encoded and an information-outage event is detected. Thus, the length of the codeword is still taken into account by the simulation. The performance with the accelerated simulation remains close to that achieved with the actual turbo code, and this behavior is due to the capacity-approaching behavior of a turbo code. The information-outage probability could also be used to predict the performance of other capacity-approaching codes, such as low-density parity check (LDPC) codes, and could be used to simulate newer standards such as LTE and WiMAX.

\section{REFERENCES}

[1] European Telecommunications Standards Institute, "Universal mobile telecommunications system (UMTS): Multiplexing and channel coding (FDD)," ETSI TS 25.212 version 6.6.0, Sept. 2005.

[2] — - "Universal mobile telecommunications system (UMTS): User equipment (UE) radio transmission and reception (FDD)," ETSI TS 25.101 version 6.9.0, Sept. 2005.

[3] — , "Universal mobile telecommunications system (UMTS); radio transmission and reception (FDD)," ETSI TS 25.104 version 6.6.0, Sept. 2005.

[4] L. Ozarow, S. Shamai, and A. D. Wyner, "Information theoretic considerations for cellular mobile radio," IEEE Trans. Veh. Tech., vol. 43, pp. 359-378, May 1994.

[5] E. Biglieri, J. Proakis, and S. Shamai, "Fading channels: Informationtheoretic and communications aspects," IEEE Trans. Inform. Theory, vol. 44, pp. 2619-2692, Oct. 1998.

[6] G. Caire and K. R. Kumar, "Information theoretic foundations of adaptive coded modulation," Proceedings of the IEEE, vol. 95, pp. 2274 2298, Dec. 2007.

[7] G. Caire, G. Taricco, and E. Biglieri, "Bit-interleaved coded modulation," IEEE Trans. Inform. Theory, vol. 44, pp. 927-946, May 1998.

[8] A. J. Viterbi, "An intuitive justification and a simplified implemetation of the MAP decoder for convolutional codes," IEEE J. Select. Areas Commun., vol. 16, no. 2, pp. 260-264, Feb. 1998.

[9] R. L. Bogusch, "Digital communications in fading channels: Modulation and coding," Mission Research Corporation, Report for AFWL, AFWLTR-87-52, Tech. Rep., April 1989.

[10] P. Hoeher, "A statistical discrete-time model for the WSSUS multipath channel," IEEE Trans. Veh. Tech., vol. 41, no. 4, pp. 461-468, May 1992.

[11] R. A. Dana, "Statistics of sampled Rician fading," Mission Research Corporation, DNA-TR-92-98, Tech. Rep., (Revised) March 2000.

[12] L. Couch, Digital and Analog Communication Systems, 5th ed. Prentice Hall, 1997.

[13] T. Ghanim and M. C. Valenti, "The throughput of hybrid-ARQ in block fading under modulation constraints," in Conf. on Information Sciences and Systems (CISS), Princeton, NJ, Mar. 2006. 